\title{
UPPER BOUNDS ON THE DIAMETER FOR FINSLER MANIFOLDS WITH WEIGHTED RICCI CURVATURE
}

\author{
Y. SOYLU
}

Received 05 January, 2018

\begin{abstract}
In this paper we obtain some Cheeger-Gromov-Taylor type compactness theorems for a forward complete and connected Finsler manifold of dimensional $n \geq 2$ via weighted Ricci curvatures. The proofs are based on the index form of a minimal unit speed geodesic segment, Bochner-Weitzenböck formula and Hessian comparison theorem.
\end{abstract}

2010 Mathematics Subject Classification: 53C60; 53B40

Keywords: diameter estimate, distortion, Finsler manifold, $S$-curvature, weighted Ricci curvature

\section{INTRODUCTION AND MAIN THEOREMS}

In [8], Myers obtained a compactness theorem in Riemannian manifolds. The theorem of Myers concludes that if Ric $\geq(n-1) K>0$, then $\operatorname{diam}(M) \leq \pi / \sqrt{K}$. Later, Cheeger-Gromov-Taylor [3] proved that if there exist $p \in M$ and $r_{0}, v>0$ such that

$$
\text { Ric } \geq(n-1) \frac{\left(\frac{1}{4}+v^{2}\right)}{r^{2}}
$$

holds for all $r(x) \geq r_{0}>0$ where $r$ is distance function defined with respect to a fixed point $p \in M$, i.e., $r(x)=d(x, p)$, then $M$ is compact and the diameter is bounded from above by $\operatorname{diam}_{p}(M)<r_{0} e^{\pi / \nu}$. By using Bakry-Emery Ricci tensor, Ric $f=$ Ric $+\operatorname{Hess} f$, Soylu [12] attained a generalization of Cheeger-Gromov-Taylor's compactness theorem.

For $m$-Bakry-Emery Ricci tensor, Wang [14] proved that, if the following inequality

$$
\operatorname{Ric}_{f, m}=\operatorname{Ric}+\operatorname{Hess} f-\frac{\mathrm{d} f \otimes \mathrm{d} f}{m-n} \geq-(m-1) \frac{K_{0}}{(1+r)^{2}}
$$

holds for all $x \in M$, where $K_{0}<-\frac{1}{4}$ and $r$ is distance function defined with respect to a fixed point $p \in M$, then $M$ is compact and the diameter has the upper bound $\operatorname{diam}(M)<2\left(e^{2 \pi / \bar{K}}-1\right)$, where $\bar{K}=\sqrt{-K_{0}-\frac{1}{4}}$.

We can find various kinds of generalizations of the Myers theorem in $[4,6,7,13$, 15].

(c) 2018 Miskolc University Press 
Finsler geometry is a natural generalization of Riemannian geometry. The validity of the Myers compactness theorem for Finsler manifolds was shown by Shen [11] without any modification. Later, using the weighted Ricci curvature $\operatorname{Ric}_{N}:=$ Ric $+\dot{S}-\frac{S^{2}}{N-n} \geq K>0, N \in(n, \infty)$, Ohta [9] obtained a compactness theorem and gave an upper bound for the diameter of $n$-dimensional Finsler manifolds as $\operatorname{diam}(M) \leq \pi \sqrt{(N-1) / K}$. In [16], Wu establish a generalized Myers theorem under line integral curvature bound for Finsler manifolds. In [2], Anastasiei extended to Finsler manifolds the compactness theorems of Ambrose and Galloway (see [1] and [5], respectively). Yin [18] acquired two Myers-type compactness theorems for a Finsler manifold with a positive weighted Ricci curvature bound and an advisable condition on the distortion or the $S$-curvature.

Throughout this paper, $(M, F)$ is a connected forward complete $n$-dimensional smooth Finsler manifold, $r(x)=d(x, p)$ is the forward distance function from $p \in M$ and $d \mu$ is an arbitrary positive $e^{\infty}$-measure on $M$. Here, there is no canonical measure like the volume measure in Riemannian geometry. Thus we begin with an arbitrary measure on $M$.

We are now ready to give our main results.

Theorem 1. Let $(M, F, d \mu)$ be a forward complete and connected Finsler manifold of dimension $n$ with arbitrary volume form and let $r$ be the distance function $r(x)=d(x, p)$ with respect to a fixed point $p \in M$. Assume that the weighted Ricci curvature

$$
\operatorname{Ric}_{\infty}:=\operatorname{Ric}+\dot{S} \geq(n-1) \frac{H}{r^{2}},
$$

and the distortion $|\tau| \leq(n-1) k$ for all $x \in M$ such that $r(x) \geq r_{0}>0$, where the constants $k$ and $H$ satisfy the inequalities $k \geq 0$ and $H>1 / 4$. Then $M$ is compact and the diameter from the point $p \in M$ satisfies

$$
\operatorname{diam}_{p}(M) \leq r_{0} \exp \left(\frac{2}{4 H-1} \sqrt{32 k^{2}+(4 H-1) \pi^{2}+16 k \sqrt{4 k^{2}+(4 H-1) H \pi^{2}}}\right) .
$$

The distortion $\tau$ is a smooth function on $M$ when $M$ is a Riemannian manifold. Therefore the diameter estimate (1.4) of Theorem 1 coincides with the diameter estimate of Theorem 1.1 in [12].

Theorem 2. Let $(M, F, d \mu)$ be a forward complete and connected Finsler manifold of dimension $n$ with arbitrary volume form and let $r$ be the distance function $r(x)=d(x, p)$ with respect to a fixed point $p \in M$. Assume that the weighted Ricci curvature

$$
\operatorname{Ric}_{N}:=\operatorname{Ric}_{\infty}-\frac{S^{2}}{N-n} \geq(N-1) \frac{H}{r^{2}}
$$

for all $N \in(n, \infty)$ and $r(x) \geq r_{0}>0$, where $H>1 / 4$. Then $M$ is compact and the diameter from the point $p \in \bar{M}$ satisfies

$$
\operatorname{diam}_{p}(M) \leq r_{0} e^{2 \pi / \sqrt{4 H-1}} .
$$


The diameter estimate (1.6) obtained in the above theorem coincides with the result of Cheeger-Gromov-Taylor in [3] obtained for the original Ricci tensor in the Riemannian manifolds.

Theorem 3. Let $(M, F, d \mu)$ be a forward complete and connected Finsler manifold of dimension $n$ with arbitrary volume form and let $r$ be the distance function $r(x)=d(x, p)$ with respect to a fixed point $p \in M$. Suppose that the weighted Ricci curvature

$$
\operatorname{Ric}_{N}:=\operatorname{Ric}_{\infty}-\frac{S^{2}}{N-n} \geq(N-1) \frac{H}{(1+r)^{2}}
$$

for all $x \in M$ and $N \in(n, \infty)$, where $H>1 / 4$. Then $M$ is compact and the diameter satisfies

$$
\operatorname{diam}(M) \leq(1+\lambda)\left(e^{2 \pi / \sqrt{4 H-1}}-1\right),
$$

where $\lambda$ is the reversibility.

We review below some basic informations about the Finsler manifolds to be used in the proofs of main theorems.

\section{A BRIEF REVIEW OF FINSLER GEOMETRY}

Let $(M, F)$ be a Finsler $n$-manifold with Finsler metric $F: T M \rightarrow[0, \infty)$. Let $\pi: T M \rightarrow M$ be the natural projection and $(x, y)$ be a point of $T M$ such that $x \in M$ and $y \in T_{x} M$. A Finsler metric is a $\bigodot^{\infty}$-Finsler structure of $M$ with the following properties:

1. $F$ is $\bigodot^{\infty}$ on $T M \backslash 0$ (Regularity),

2. $F(x, \lambda y)=\lambda F(x, y)$ for all $\lambda>0$ (Positive homogeneity),

3. The $n \times n$ Hessian matrix

$$
g_{i j}:=\frac{1}{2}\left[F^{2}\right]_{y^{i} y^{j}}
$$

is positive-definite at every point of $T M \backslash 0$ (Strong convexity).

The Chern curvature $R^{V}$ for vectors fields $X, Y, Z \in T_{x} M \backslash 0$ is defined by

$$
R^{V}(X, Y) Z:=\nabla_{X}^{V} \nabla_{Y}^{V} Z-\nabla_{Y}^{V} \nabla_{X}^{V} Z-\nabla_{[X, Y]}^{V} Z,
$$

and the flag curvature is defined as follows:

$$
K(V, W):=\frac{g_{V}\left(R^{V}(V, W) W, V\right)}{g_{V}(V, V) g_{V}(W, W)-g_{V}(V, W)^{2}},
$$

where $V, W \in T_{x} M \backslash 0$ are linearly independent vectors. Then the Ricci curvature of $V$ (as the trace of the flag curvature) is defined by

$$
\operatorname{Ric}(V):=\sum_{i=1}^{n-1} K\left(V, E_{i}\right),
$$


where $\left\{E_{1}, E_{2}, \ldots, E_{n-1}, V / F(V)\right\}$ is an orthonormal basis of $T_{x} M$ with respect to $g_{V}$. 0 ,

Let $d \mu=\sigma(x) d x^{1} d x^{2} \ldots d x^{n}$ be the volume form on $M$. For a vector $V \in T_{x} M \backslash$

$$
\tau(x, V):=\ln \frac{\sqrt{\operatorname{det}\left(g_{i j}(x, V)\right)}}{\sigma(x)}
$$

is a scalar function on $T_{x} M \backslash 0$ which is called the distortion of $(M, F, d \mu)$. We say that the distortion $\tau$ is a $\mathcal{C}^{\infty}$-function, if $M$ is a Riemannian manifold. Setting

$$
S(x, V):=\left.\frac{d}{d t}(\tau(\gamma(t), \dot{\gamma}(t)))\right|_{t=0},
$$

where $\gamma$ is the geodesic with $\gamma(0)=x, \dot{\gamma}(0)=V . S(x, \lambda V)=\lambda S(x, V)$ for all $\lambda>0 . S$ is a scalar function on $T_{x} M \backslash 0$ which is called the $S$-curvature. From the definition, it seems that the $S$-curvature measures the rate of change in the distortion along geodesics in the direction $V \in T_{x} M$.

For all $N \in(n, \infty)$, we define the weighted Ricci curvature of $(M, F, d \mu)$ as follows (see [9]):

$$
\left\{\begin{array}{l}
\operatorname{Ric}_{N}(V):=\operatorname{Ric}(V)+\dot{S}(V)-\frac{S(V)^{2}}{N-n}, \\
\operatorname{Ric}_{\infty}(V):=\operatorname{Ric}(V)+\dot{S}(V), \\
\operatorname{Ric}_{n}(V):= \begin{cases}\operatorname{Ric}+\dot{S}(V), & \text { if } S(V)=0 \\
-\infty & \text { otherwise. }\end{cases}
\end{array}\right.
$$

Also $\operatorname{Ric}_{N}(c V):=c^{2} \operatorname{Ric}_{N}(V)$ for $c>0$.

We say that $(M, F)$ is forward complete if each geodesic $\gamma:[0, \ell] \rightarrow M$ is extended to a geodesic on $[0, \infty)$, in other words, if exponential map is defined on whole $T M$. Then the Hopf-Rinow theorem gives that every pair of points in $M$ can be joined by a minimal geodesic.

The Legendre transformation $\mathfrak{L}: T M \rightarrow T^{*} M$ is defined by

$$
\mathfrak{L}(W):= \begin{cases}g_{W}(W, .), & W \neq 0 \\ 0 & W=0 .\end{cases}
$$

For a smooth function $h: M \rightarrow \mathbb{R}$, the gradient vector of $h$ at $x \in M$ is defined as $\nabla h(x):=\mathfrak{L}^{-1}(d h)$.

Given a smooth vector field $Z=Z^{i} \partial / \partial x^{i}$ on $M$, the divergence of $Z$ with respect to an arbitrary volume form $d \mu=e^{\varphi} d x^{1} d x^{2} \ldots d x^{n}$ is defined by

$$
\operatorname{div} Z:=\sum_{i=1}^{n}\left(\frac{\partial Z^{i}}{\partial x^{i}}+Z^{i} \frac{\partial \varphi}{\partial x^{i}}\right) .
$$

Then we define the Finsler-Laplacian of $h$ by $\Delta h:=\operatorname{div}(\nabla h)=\operatorname{div}\left(\mathfrak{L}^{-1}(d h)\right)$.

The following lemma is useful to prove Theorem 3 (see [17]). 
Lemma 1. Let $(M, F, d \mu)$ be a Finsler n-manifold, and $h: M \rightarrow \mathbb{R}$ a smooth function on $M$. Then on $U=\left\{x \in M:\left.\nabla h\right|_{x} \neq 0\right\}$ we have

$$
\Delta h=\sum_{i} H(h)\left(E_{i}, E_{i}\right)-S(\nabla h):=\operatorname{tr}_{\nabla h} H(h)-S(\nabla h),
$$

where $E_{1}, E_{2}, \ldots, E_{n}$ is a local $g_{\nabla h}$-orthonormal frame on $U$.

Finally, define reversibility $\lambda:=\lambda(M, F)$ as follows:

$$
\lambda:=\sup _{x \in M, y \in T M \backslash 0} \frac{F(x,-y)}{F(x, y)} .
$$

Obviously, $\lambda \in[1, \infty]$, and $\lambda=1$ if and only if $(M, F)$ is reversible.

\section{THE PROOFS OF THE THEOREMS}

Let $(M, F, d \mu)$ be a Finsler manifold of dimensional $n$ and $r(x)=d(x, p)$ be a distance function with respect to a fixed point $p \in M$. It is well known that $r$ is only smooth on $M-\left(C_{p} \cup\{p\}\right)$ where $C_{p}$ is the cut locus of the point $p \in M$. We assume that $\gamma$ is a minimal unit speed geodesic segment. We have $\nabla r=\dot{\gamma}$ in the adapted coordinates with respect to the $r$, and also have $F(\nabla r)=1$ (see [11]). On the other hand, using the Finsler metric we obtain a weighted Riemannian metric $g_{\nabla r}$. Thus we can apply the Riemannian calculation for $g_{\nabla r}$ (on $M-\left(C_{p} \cup\{p\}\right)$ ).

In order to prove the Theorem 1 and Theorem 2, we use the index form of a minimal unit speed geodesic, and to prove Theorem 3, we use Bochner-Weitzenböck formula and Hessian comparison theorem in Finsler geometry.

Proof of Theorem 1. Let $q \in M$ be a point and let $\sigma$ be a minimal unit speed geodesic segment from $p$ to $q$ of length $\ell$ such that $\sigma(0)=p, \sigma(\ell)=q$ and $\ell>r_{0}>$ 0 . Since the inequality $\ell>r_{0}$ holds, $\ell$ can be parametrized by $\mu>0$ such that

$$
\ell=r_{0} e^{\mu \pi}>r_{0} .
$$

By virtue of any subsegment of a minimal unit speed geodesic segment is also a minimal unit speed geodesic segment, we have the minimal unit speed geodesic segment $\gamma$ defined by $\gamma(t)=\left.\sigma\right|_{\left[r_{0}, \ell\right]}(t)$ where $\gamma:\left[r_{0}, \ell\right] \rightarrow M$ and $\gamma\left(r_{0}\right)=\sigma\left(r_{0}\right)=\tilde{q}$, $\gamma(\ell)=\sigma(\ell)=q$. Let $\left\{E_{1}=\dot{\gamma}, E_{2}, \ldots, E_{n}\right\}$ be a parallel $g_{\nabla r}$-orthonormal frame along $\gamma$ and let $f \in \mathcal{C}^{\infty}\left(\left[r_{0}, \ell\right]\right)$ be a real-valued smooth function such that $f\left(r_{0}\right)=$ $f(\ell)=0$. Then we have

$$
\mathrm{I}\left(f E_{i}, f E_{i}\right)=\int_{r_{0}}^{\ell}\left(g_{\nabla r}\left(\dot{f} E_{i}, \dot{f} E_{i}\right)-g_{\nabla r}\left(R^{\nabla r}\left(f E_{i}, \nabla r\right) \nabla r, f E_{i}\right)\right) d t .
$$


It is obvious that (3.2) yields, by $g_{\nabla r}\left(R^{\nabla r}(\nabla r, \nabla r) \nabla r, \nabla r\right)=0$ and the assumption (1.3) given in Theorem 1,

$$
\begin{aligned}
\sum_{i=2}^{n} \mathrm{I}\left(f E_{i}, f E_{i}\right) & =\int_{r_{0}}^{\ell}\left((n-1) \dot{f}^{2}-f^{2} \operatorname{Ric}(\nabla r)\right) d t \\
& =\int_{r_{0}}^{\ell}\left((n-1) \dot{f}^{2}-f^{2} \operatorname{Ric}_{\infty}(\nabla r)+f^{2} \dot{S}(\nabla r)\right) d t \\
& \leq \int_{r_{0}}^{\ell}\left((n-1)\left(\dot{f}^{2}-\frac{H f^{2}}{r^{2}}\right)+f^{2} \dot{S}(\nabla r)\right) d t
\end{aligned}
$$

Here, the term $f^{2} \dot{S}(\nabla r)$ equals to

$$
\begin{aligned}
f^{2} \dot{S}(\nabla r) & \left.=-2 f \dot{f} S(\nabla r)+\frac{d}{d t}\left(f^{2} S(\nabla r)\right)\right)=-2 f \dot{f} \frac{d \tau}{d t}+\frac{d}{d t}\left(f^{2} S(\nabla r)\right) \\
& \left.=2 \tau \frac{d}{d t}(f \dot{f})-2 \frac{d}{d t}(\tau f \dot{f})+\frac{d}{d t}\left(f^{2} S(\nabla r)\right)\right) .
\end{aligned}
$$

Integrating both sides of (3.4) and using the assumption $|\tau| \leq(n-1) k$, we obtain

$$
\int_{r_{0}}^{\ell}\left(f^{2} \dot{S}(\nabla r)\right) d t=2 \int_{r_{0}}^{\ell} \tau \frac{d}{d t}(f \dot{f}) d t \leq 2(n-1) k \int_{r_{0}}^{\ell}\left|\frac{d}{d t}(f \dot{f})\right| d t,
$$

because of $f\left(r_{0}\right)=f(\ell)=0$. By use of (3.5), the inequality (3.3) becomes

$$
\sum_{i=2}^{n} \mathrm{I}\left(f E_{i}, f E_{i}\right) \leq \int_{r_{0}}^{\ell}(n-1)\left(\dot{f}^{2}-\frac{H f^{2}}{r^{2}}\right) d t+2(n-1) k \int_{r_{0}}^{\ell}\left|\frac{d}{d t}(f \dot{f})\right| d t .
$$

Set

$$
f(t)=\mu r_{0} \sqrt{r(\gamma(t))} \sin \left(\frac{1}{\mu} \ln \frac{r(\gamma(t))}{r_{0}}\right) .
$$

Therefore we have

$$
\begin{aligned}
\frac{1}{r_{0}^{2}(n-1)} \sum_{i=2}^{n} \mathrm{I}\left(f E_{i}, f E_{i}\right) \leq & -\frac{1}{4} \int_{r_{0}}^{\ell} \frac{(4 H-1) \mu^{2}}{r} \sin ^{2}\left(\frac{1}{\mu} \ln \frac{r}{r_{0}}\right) d r \\
& +\int_{r_{0}}^{\ell} \frac{1}{r}\left(\cos ^{2}\left(\frac{1}{\mu} \ln \frac{r}{r_{0}}\right)+\frac{\mu}{2} \sin \left(\frac{2}{\mu} \ln \frac{r}{r_{0}}\right)\right) d r \\
& +2 k \int_{r_{0}}^{\ell} \frac{1}{r}\left|\frac{\mu}{2} \sin \left(\frac{2}{\mu} \ln \frac{r}{r_{0}}\right)+\cos \left(\frac{2}{\mu} \ln \frac{r}{r_{0}}\right)\right| d r .
\end{aligned}
$$

In (3.8), considering the change variable $u=\ln \frac{r}{r_{0}}$, by $\ell=r_{0} e^{\mu \pi}$, we obtain

$$
\frac{1}{r_{0}^{2}(n-1)} \sum_{i=2}^{n} \mathrm{I}\left(f E_{i}, f E_{i}\right) \leq-\frac{1}{4} \int_{0}^{\mu \pi}(4 H-1) \mu^{2} \sin ^{2}\left(\frac{1}{\mu} u\right) d u
$$




$$
\begin{aligned}
& +\int_{0}^{\mu \pi}\left(\cos ^{2}\left(\frac{1}{\mu} u\right)+\frac{\mu}{2} \sin \left(\frac{2}{\mu} u\right)\right) d u \\
& +2 k \int_{0}^{\mu \pi}\left|\frac{\mu}{2} \sin \left(\frac{2}{\mu} u\right)+\cos \left(\frac{2}{\mu} u\right)\right| d u,
\end{aligned}
$$

from which

$$
\frac{1}{r_{0}^{2}(n-1)} \sum_{i=2}^{n} \mathrm{I}\left(f E_{i}, f E_{i}\right) \leq \frac{\mu}{8}\left(4 \pi-(4 H-1) \pi \mu^{2}+16 k \sqrt{\mu^{2}+4}\right) .
$$

In the right hand side of (3.10), if the inequality

$$
4 \pi-(4 H-1) \pi \mu^{2}+16 k \sqrt{\mu^{2}+4}<0
$$

holds, then the index form $I$ is not positive semi-definite. This is a contradiction. Hence, we must take

$$
4 \pi-(4 H-1) \pi \mu^{2}+16 k \sqrt{\mu^{2}+4} \geq 0 .
$$

Thus

$$
\mu \leq \frac{2}{(4 H-1) \pi} \sqrt{32 k^{2}+(4 H-1) \pi^{2}+16 k \sqrt{4 k^{2}+(4 H-1) H \pi^{2}}} .
$$

Using the parametrization $\ell=r_{0} e^{\mu \pi}$ given in (3.1), we find

$$
\ell=r_{0} e^{\mu \pi} \leq r_{0} \exp \left(\frac{2}{4 H-1} \sqrt{\left.32 k^{2}+(4 H-1) \pi^{2}+16 k \sqrt{4 k^{2}+(4 H-1) H \pi^{2}}\right) .}\right.
$$

Thus, $M$ is compact and the diameter of $M$ has the upper bound (1.4).

Proof of Theorem 2. By similar arguments given in the proof of Theorem 1, we have

$$
\sum_{i=2}^{n} \mathrm{I}\left(f E_{i}, f E_{i}\right)=\int_{r_{0}}^{\ell}\left((n-1) \dot{f}^{2}-f^{2} \operatorname{Ric}(\nabla r)\right) d t .
$$

Using the assumption (1.5) in the above integral expression, we get

$$
\begin{aligned}
\sum_{i=2}^{n} \mathrm{I}\left(f E_{i}, f E_{i}\right) \leq & \int_{r_{0}}^{\ell}\left((n-1) \dot{f}^{2}-(N-1) \frac{H f^{2}}{r^{2}}\right) d t \\
& +\int_{r_{0}}^{\ell}\left(f^{2} \dot{S}(\nabla r)-f^{2} \frac{(S(\nabla r))^{2}}{N-n}\right) d t .
\end{aligned}
$$

In the inequality (3.16), the term $f^{2} \dot{S}(\nabla r)$ equals to

$$
f^{2} \dot{S}(\nabla r)=-2 f \dot{f} S(\nabla r)+\frac{d}{d t}\left(f^{2} S(\nabla r)\right) .
$$


Integrating both sides of (3.17), we obtain

$$
\int_{r_{0}}^{\ell} f^{2} \dot{S}(\nabla r) d t=\int_{r_{0}}^{\ell}-2 f \dot{f} S(\nabla r) d t
$$

by $f\left(r_{0}\right)=f(\ell)=0$. If we take $P=-\dot{f}$ and $T=f S(\nabla r)$, then the CauchySchwarz inequality

$$
\int_{r_{0}}^{\ell} P T d t=\int_{r_{0}}^{\ell}-f \dot{f} S(\nabla r) d t \leq\left(\int_{r_{0}}^{\ell} \dot{f}^{2} d t\right)^{1 / 2}\left(\int_{r_{0}}^{\ell} f^{2}(S(\nabla r))^{2} d t\right)^{1 / 2} .
$$

Because of the facts

$$
A=(N-n) \int_{r_{0}}^{\ell} \dot{f}^{2} d t \geq 0 \text { and } B=\frac{1}{N-n} \int_{r_{0}}^{\ell} f^{2}(S(\nabla r))^{2} d t \geq 0,
$$

where $N \in(n, \infty)$, we have the inequality $\sqrt{A B} \leq \frac{1}{2}(A+B)$, i.e.,

$$
\begin{aligned}
\left(\int_{r_{0}}^{\ell} \dot{f}^{2} d t\right)^{1 / 2}\left(\int_{r_{0}}^{\ell} f^{2}(S(\nabla r))^{2} d t\right)^{1 / 2} \leq & \int_{r_{0}}^{\ell} \frac{1}{2}(N-n) \dot{f}^{2} d t \\
& +\int_{r_{0}}^{\ell} f^{2} \frac{(S(\nabla r))^{2}}{2(N-n)} d t .
\end{aligned}
$$

Using (3.21) in (3.19), we find

$$
\int_{r_{0}}^{\ell}-f \dot{f} S(\nabla r) d t \leq \int_{r_{0}}^{\ell}\left(\frac{1}{2}(N-n) \dot{f}^{2}+f^{2} \frac{(S(\nabla r))^{2}}{2(N-n)}\right) d t .
$$

Therefore we have

$$
\int_{r_{0}}^{\ell} f^{2} \dot{S}(\nabla r) d t=\int_{r_{0}}^{\ell}-2 f \dot{f} S(\nabla r) d t \leq \int_{r_{0}}^{\ell}\left((N-n) \dot{f}^{2}+f^{2} \frac{(S(\nabla r))^{2}}{N-n}\right) d t .
$$

Inserting (3.23) into (3.16), we obtain

$$
\sum_{i=2}^{n} \mathrm{I}\left(f E_{i}, f E_{i}\right) \leq(N-1) \int_{r_{0}}^{\ell}\left(\dot{f}^{2}-\frac{H f^{2}}{r^{2}}\right) d t .
$$

In the inequality (3.24), let us consider the choice

$$
f(t)=\mu r_{0} \sqrt{r(\gamma(t))} \sin \left(\frac{1}{\mu} \ln \frac{r(\gamma(t))}{r_{0}}\right) .
$$

Thereby the inequality (3.24) yields

$$
\frac{1}{N-1} \sum_{i=2}^{n} \mathrm{I}\left(f E_{i}, f E_{i}\right) \leq \int_{r_{0}}^{\ell} \frac{r_{0}^{2}}{r}\left(\cos ^{2}\left(\frac{1}{\mu} \ln \frac{r}{r_{0}}\right)+\frac{\mu}{2} \sin \left(\frac{2}{\mu} \ln \frac{r}{r_{0}}\right)\right) d r
$$




$$
-\frac{1}{4} \int_{r_{0}}^{\ell} \frac{r_{0}^{2}}{r}(4 H-1) \mu^{2} \sin ^{2}\left(\frac{1}{\mu} \ln \frac{r}{r_{0}}\right) d r .
$$

In the above inequality, considering the change variable $u=\ln \frac{r}{r_{0}}$, , by use of $\ell=$ $r_{0} e^{\mu \pi}$, we get

$$
\begin{aligned}
\frac{1}{N-1} \sum_{i=2}^{n} \mathrm{I}\left(f E_{i}, f E_{i}\right) \leq & \int_{0}^{\mu \pi} r_{0}^{2}\left(\cos ^{2}\left(\frac{1}{\mu} u\right)+\frac{\mu}{2} \sin \left(\frac{2}{\mu} u\right)\right) d u \\
& -\frac{1}{4} \int_{0}^{\mu \pi} r_{0}^{2}(4 H-1) \mu^{2} \sin ^{2}\left(\frac{1}{\mu} u\right) d u,
\end{aligned}
$$

which implies

$$
\frac{1}{N-1} \sum_{i=2}^{n} \mathrm{I}\left(f E_{i}, f E_{i}\right) \leq r_{0}^{2} \frac{\mu \pi}{8}\left(4-(4 H-1) \mu^{2}\right) .
$$

In the right hand side of (3.28), if the inequality

$$
4-(4 H-1) \mu^{2}<0
$$

holds, then we conclude that the index form $I$ is not positive semi-definite. But, since $\gamma$ is minimal geodesic, this is a contradiction. Hence, we must take

$$
4-(4 H-1) \mu^{2} \geq 0 \text {. }
$$

Thus we obtain

$$
\mu \leq \frac{2}{\sqrt{4 H-1}} .
$$

Using the parametrization $\ell=r_{0} e^{\mu \pi}$, we find

$$
\ell=r_{0} e^{\mu \pi} \leq r_{0} e^{2 \pi / \sqrt{4 H-1}} .
$$

Thus, $M$ is compact and the diameter of $M$ has the upper bound (1.6).

Proof of Theorem 3. We know that $r(x)=d(x, p)$ is a distance function from a fixed point $p \in M$ and it is smooth on $M-\left(C_{p} \cup\{p\}\right)$. Also it satisfies $F(\nabla r)=1$. In Finsler geometry, recall that the Bochner-Weitzenböck formula [10] for a smooth function $u \in \mathcal{C}^{\infty}(M)$

$$
0=\Delta^{\nabla u}\left(\frac{F(\nabla u)^{2}}{2}\right)=\operatorname{Ric}_{\infty}(\nabla u)+D(\Delta u)(\nabla u)+\left\|\nabla^{2} u\right\|_{H S(\nabla u)}^{2} .
$$

From the Bochner formula applied to distance function $r$ and by Lemma 1, we have, on $M-\left(C_{p} \cup\{p\}\right)$,

$$
\begin{aligned}
0 & =\operatorname{Ric}_{\infty}(\nabla r)+D(\Delta r)(\nabla r)+\left\|\nabla^{2} r\right\|_{H S(\nabla r)}^{2} \\
& \geq \operatorname{Ric}_{\infty}(\nabla r)+g_{\nabla r}(\nabla \nabla r \Delta r, \nabla r)+\frac{(\Delta r+S(\nabla r))^{2}}{n-1} .
\end{aligned}
$$


By virtue of the inequality $(a \mp b)^{2} \geq \frac{1}{\beta+1} a^{2}-\frac{1}{\beta} b^{2}$ holding for all real numbers $a, b$ and positive real number $\beta$, we have

$$
\frac{(\Delta r+S(\nabla r))^{2}}{n-1} \geq \frac{(\Delta r)^{2}}{(n-1)(\beta+1)}-\frac{(S(\nabla r))^{2}}{(n-1) \beta} .
$$

In the case where $N>n$, taking $\beta=\frac{N-n}{n-1}>0$, (3.34) yields

$$
0 \geq \operatorname{Ric}_{\infty}(\nabla r)+g_{\nabla r}\left(\nabla^{\nabla r} \Delta r, \nabla r\right)+\frac{(\Delta r)^{2}}{N-1}-\frac{(S(\nabla r))^{2}}{N-n} .
$$

Applying the assumption (1.7) given in Theorem 3 to (3.36), we find

$$
0 \geq \partial_{r}(\Delta r)+\frac{(\Delta r)^{2}}{N-1}+(N-1) \frac{H}{(1+r)^{2}} .
$$

The above inequality can be rewritten as

$$
0 \geq \partial_{r}\left(\frac{\Delta r}{N-1}\right)+\left(\frac{\Delta r}{N-1}\right)^{2}+\frac{H}{(1+r)^{2}} .
$$

We know from the Hessian comparison theorem in [17], if there is a local vector field $X$ on an open set $U$ of $p \in M$ with $g_{\nabla r}(X, X)=1, g_{\nabla r}(\nabla r, X)=0$, then $H(r)(X, X) \sim \frac{1}{r}$ as $r \rightarrow 0^{+}$. Hence, using the Lemma 1, we have

$$
\lim _{r \rightarrow 0^{+}} r\left(\frac{1}{N-1} \Delta r\right)=\lim _{r \rightarrow 0^{+}} r\left(\frac{1}{N-1}\left(\operatorname{tr}_{\nabla r} H(r)-S(\nabla r)\right)\right)=\frac{n-1}{N-1}<1,
$$

where $N>n$. By (3.38) and (3.39), we obtain, on $M-\left(C_{p} \cup\{p\}\right)$,

$$
\frac{1}{N-1} \Delta r \leq \frac{1}{2(1+r)}\left(1+\sqrt{4 H-1} \cot \left(\frac{\sqrt{4 H-1}}{2} \ln (1+r)\right)\right),
$$

where $H>1 / 4$. Indeed, the function

$$
Y(r)=\frac{1}{2(1+r)}\left(1+\sqrt{4 H-1} \cot \left(\frac{\sqrt{4 H-1}}{2} \ln (1+r)\right)\right)
$$

is a solution of the Riccati differential equation

$$
Y^{\prime}(r)+(Y(r))^{2}+\frac{H}{(1+r)^{2}}=0 .
$$

Because of $\lim _{r \rightarrow 0^{+}} r Y(r)=1$ and (3.39), we have

$$
\lim _{r \rightarrow 0^{+}} r\left(\frac{1}{N-1} \Delta r\right) \leq \lim _{r \rightarrow 0^{+}} r Y(r) .
$$

Thus, for a sufficiently small positive constant $\varepsilon \in(0, T)$ the inequality

$$
\frac{1}{N-1} \Delta r(\varepsilon) \leq Y(\varepsilon)
$$


is ensured. In that case, the Riccati comparison theorem gives the inequality

$$
\frac{1}{N-1} \Delta r(t) \leq Y(t)
$$

for every $t \in[\varepsilon, T)$.

Let $q \in M$ be any point, and let $\sigma$ be a minimal unit speed geodesic segment from $p$ to $q$. Suppose that the inequality

$$
d(p, q)>e^{2 \pi / \sqrt{4 H-1}}-1
$$

is satisfied. Then, since $\sigma$ is a minimal unit speed geodesic segment from $p$ to $q$, we have the fact that the point $\sigma\left(e^{2 \pi / \sqrt{4 H-1}}-1\right)$ is outside the cut locus of $p \in M$, i.e.,

$$
\sigma\left(e^{2 \pi / \sqrt{4 H-1}}-1\right) \in M-\left(C_{p} \cup\{p\}\right) .
$$

Therefore the distance function $r$ is smooth at this point. Namely, at this point, left hand side of (3.40) is a constant. However, the right side of (3.40) tends to $-\infty$ as $r \rightarrow\left(e^{2 \pi / \sqrt{4 H-1}}-1\right)^{-}$, i.e.,

$$
\lim _{r \rightarrow\left(e^{2 \pi / \sqrt{4 H-1}}-1\right)^{-}} \frac{1}{2(1+r)}\left(1+\sqrt{4 H-1} \cot \left(\frac{\sqrt{4 H-1}}{2} \ln (1+r)\right)\right)=-\infty .
$$

This is a contradiction. Hence, (3.46) does not hold. It must be

$$
d(p, q) \leq e^{2 \pi / \sqrt{4 H-1}}-1 .
$$

Therefore $M$ is compact. Let $\lambda$ be the reversibility. For any points $p^{\prime}, q^{\prime} \in M$, due to the triangle inequality and the inequality (3.49), we obtain

$$
d\left(p^{\prime}, q^{\prime}\right) \leq d\left(p^{\prime}, p\right)+d\left(p, q^{\prime}\right) \leq \lambda d\left(p, p^{\prime}\right)+d\left(p, q^{\prime}\right),
$$

and so

$$
d\left(p^{\prime}, q^{\prime}\right) \leq(1+\lambda)\left(e^{2 \pi / \sqrt{4 H-1}}-1\right)
$$

This completes the proof of theorem.

\section{REFERENCES}

[1] W. Ambrose, “A theorem of Myers," Duke Math. J, vol. 24, no. 3, pp. 345-348, 1957, doi: 10.1215/S0012-7094-57-02440-7.

[2] M. Anastasiei, "Galloway's compactness theorem on Finsler manifolds," Balkan J. Geom. Appl, vol. 20, no. 2, pp. 1-8, 2015.

[3] J. Cheeger, M. Gromov, and M. Taylor, "Finite propagation speed, Kernel estimates for functions of the Laplace operator, and the geometry of complete Riemannian manifolds," J. Differ. Geom, vol. 17, no. 1, pp. 15-53, 1982, doi: 10.4310/jdg/1214436699.

[4] M. Fernández-López and E. García-Río, "A remark on compact Ricci solitons," Math. Ann, vol. 340, no. 4, pp. 893-896, 2008, doi: 10.1007/s00208-007-0173-4.

[5] G. J. Galloway, "A generalization of Myers theorem and an application to relativistic cosmology," J. Differ. Geom, vol. 14, no. 1, pp. 105-116, 1979, doi: 10.4310/jdg/1214434856. 
[6] M. Limoncu, "Modifications of the Ricci tensor and applications," Arch. Math, vol. 95, no. 2, pp. 191-199, 2010, doi: 10.1007/s00013-010-0150-0.

[7] M. Limoncu, "The Bakry-Emery Ricci tensor and its applications to some compactness theorems," Math. Z, vol. 271, no. 3-4, pp. 715-722, 2012, doi: 10.1007/s00209-011-0886-7.

[8] S. B. Myers, "Riemannian manifolds with positive mean curvature," Duke Math. J, vol. 8, no. 2, pp. 401-404, 1941, doi: 10.1215/S0012-7094-41-00832-3.

[9] S. Ohta, "Finsler interpolation inequalities," Calc. Var. Partial Differ. Equ, vol. 36, no. 2, pp. 211-249, 2009, doi: 10.1007/s00526-009-0227-4.

[10] S. Ohta and K. T. Sturm, "Bochner-Weitzenböck formula and Li-Yau estimates on Finsler manifolds," Adv. Math, vol. 252, pp. 429-448, 2014, doi: 10.1016/j.aim.2013.10.018.

[11] Z. Shen, Lectures on Finsler Geometry. Singapore: World Scientific, 2001.

[12] Y. Soylu, "A Myers-type compactness theorem by the use of Bakry-Emery Ricci tensor," Differ. Geom. Appl, vol. 54, pp. 245-250, 2017, doi: 10.1016/j.difgeo.2017.04.005.

[13] H. Tadano, "Remark on a diameter bound for complete Riemannian manifolds with positive Bakry-Émery Ricci curvature," Differ. Geom. Appl, vol. 44, pp. 136-143, 2016, doi: 10.1016/j.difgeo.2015.11.001.

[14] L. F. Wang, “A Myers theorem via m-Bakry-Émery curvature,” Kodai Math. J, vol. 37, no. 1, pp. 187-195, 2014, doi: 10.2996/kmj/1396008254.

[15] G. Wei and W. Wylie, "Comparison geometry for the Bakry-Emery Ricci tensor," J. Diff. Geom, vol. 83, no. 2, pp. 377-405, 2009, doi: 10.4310/jdg/1261495336.

[16] B. Wu, "A note on the generalized Myers theorem for Finsler manifolds," Bull. Korean Math. Soc, vol. 50, no. 3, pp. 833-837, 2013, doi: 10.4134/BKMS.2013.50.3.833.

[17] B. Wu and Y. Xin, "Comparison theorems in Finsler geometry and their applications," Math. Ann, vol. 337, no. 1, pp. 177-196, 2007, doi: 10.1007/s00208-006-0031-9.

[18] S. Yin, "Two compactness theorems on Finsler manifolds with positive weighted Ricci curvature," Results Math, vol. 72, no. 1-2, pp. 319-327, 2017, doi: 10.1007/s00025-017-0673-9.

Author's address

Y. Soylu

Giresun University, Department of Mathematics, 28100 Giresun, Turkey

E-mail address: yasemin.soyluegiresun.edu.tr 